\title{
Luteal Phase Support using Oral Dydrogesterone-a Prospective Treatment for Future Replacing Micronized Vaginal Progesterone
}

\author{
Kulvinder Kochar Kaur ${ }^{1 *}$, Gautam Allahbadia ${ }^{2}$ and Mandeep Singh ${ }^{3}$ \\ ${ }^{1}$ Scientific Director, Centre For Human Reproduction, Punjab, India \\ ${ }^{2}$ Department of Obstetrics and Gynecology, Mumbai, India \\ ${ }^{3}$ Department of Neurology, Swami Satyanand Hospital, Punjab, India
}

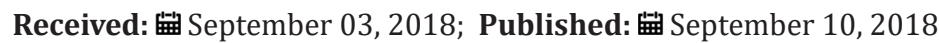

*Corresponding author: Kulvinder Kochar Kaur, Scientific Director, Centre For Human Reproduction 721, G.T.B. Nagar, Jalandhar-144001, Punjab, India

\begin{abstract}
Although micronized vaginal progesterone is the accepted norm for use in luteal phase support (LPS) in controlled ovarian stimulation (COS) that is used for in vitro fertilization (IVF) cycles, recently importance of oral Dydrogesterone has got the importance in lieu of its oral availability, cheap, no cumbersome side effects and no definitive newer fetal side effects. After the LOTUS 1 trial with a multicenter double placebo, double dummy design it has proved an equal efficacy if not superiority of oral D, over micronized vaginal progesterone and it seems D might soon become the standard of care for LPS in conventional IVF cycles besides its routine indication for recurrent abortions.
\end{abstract}

Keywords: LPS; COS; IVF; Dydrogesterone; Micronized vaginal progesterone

Abbreviations: LPS: Luteal Phase Support; COS: Controlled Ovarian Stimulation; IVF: In Vitro Fertilization; ET: Embryo Transfer

\section{Dydrogesterone(D)-Pharmacology}

A potent orally active progesterone receptor agonist D was developed in the1950's. Structurally it is 6-dehyro-9 $\beta, 10 \alpha$-progest (figure 1). It has been used for multiple disorders like premenstrual syndrome [1], cycle irregularity [2], endometriosis [3], habitual abortion [4], along with for post-menopausal hormone therapy [5], since its advent. Advantages of D and its active metabolite, 20hydroxy D, is that unlike other progesterone (P) member family, they do not have any relevant clinically, agonistic or antagonistic activity on either androgen, estrogen and glucocorticoid receptors and have only mild mineralocorticoid properties [6-8]. Main advantage from other synthetic P's is lack of androgenic side effects like hirsutism, voice changes, acne etc. and no masculinizing changes in the fetus. Because of safety concerns in view of crossreceptor activation has prevented use of a majority of progestins during fertility therapy and pregnancy. Bioidentical P,17-Hydroxy $\mathrm{P}$ caproate and dydrogesterone are the only ones found to be safe for the fetus which is growing. This D has very little effect on gonadotrophin release and hence does not interfere with follicular growth along with corpus luteum formation and maintenance. Clinically used doses of 5-30mg, they do not suppress ovulation in humans [6], although in recent times $20 \mathrm{mg} / \mathrm{d}$ of D got used as an alternative to chlormadinone acetate for preventing premature $\mathrm{LH}$ surges in the scenario of controlled ovarian stimulation (COS) [9].

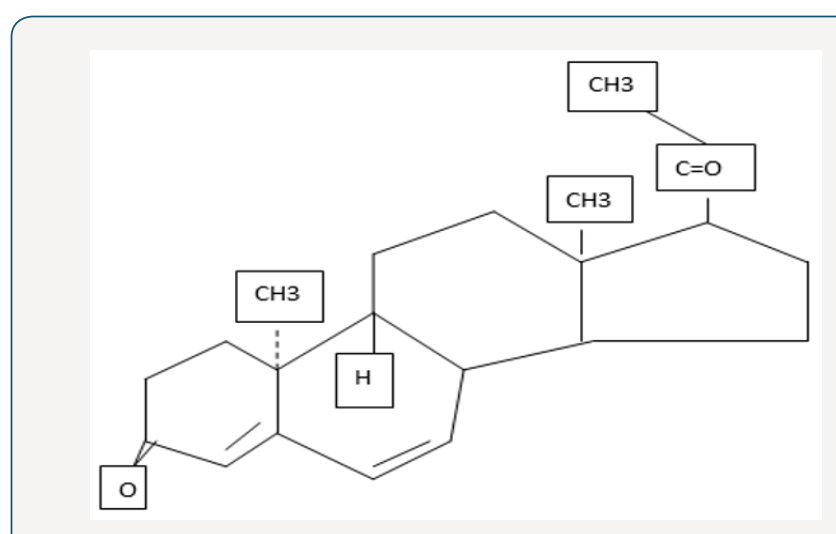

Figure 1: Dydrogesterone. 
It has good oral bioavailability(-28\%) though same is not true for the naturally occurring P. Half-life of D is $5-7 \mathrm{~h}$ and that of $20(\mathrm{OH})$ D is $14-17$ h.Prereceptor regulation of action occurs secondary to conversion of D to its biologically active $200 \mathrm{H}$ metabolite, by the enzyme aldoketo reductase 1C1[10], which is an enzyme that also converts $\mathrm{P}$ to its less potent metabolite 20-hydoxy P. D got withdrawn in USA for commercial reasons, similarly in UK in 2008,and Australian market in 2011.In USA it got licensed in 1961 ,and this license got transferred from company to company. In 1997, the new application owner Solvay, withdrew the product, as the registered indications were not commercially viable /or conflicting interests with other products were there regarding which Solvay was license holder. Low sales in UK and Australia of this cheap drug, with no new and commercially interesting indications caused its withdrawal from market.

Still currently it is licensed in more than 100 countries globally, with $>20$ european countries having atleast one reason for use in pregnancy. Commonest brand name having D tablets are duphaston (10mg tablet) and Femoston (containing D with E2 in one tablet in different doses), which is used for post-menopausal hormone treatment. D has been used as an exogenous supplement for long for the supplementation of endogenous $\mathrm{P}$ production by CL and placenta. A definitive proof of luteal phase (LPD) defect as an independent cause of infertility had never been established [11], though luteal phase defect in COS with multifollicular development as an iatrogenic phenomenon occurs which is done with the idea of doing oocyte retrieval for in vitro fertilization (IVF) [12]. In studies comparing different progestogens vs placebo, in COS IVF treatment cycles, benefit has been shown like improved pregnancy rates, live birth rates with progestogens [13]. Hence routinely progestogens get used in IVF treatment cycles.

\section{Role of D for LPS in fresh IVF Cycles}

With use of D for several years Chakravaty et al were the 1st to do systematic comparisons of oral D vs vaginal P [14-17]. He randomized 430patients of which 351 got luteal support with micronized $P(600 \mathrm{mg} / \mathrm{d})$ and 79 with oral $\mathrm{D}(20 \mathrm{mg} / \mathrm{d})$ after COS in a long GnRH agonist protocol, utilizing 10,000iu HCG for triggering. They found that delivery rates were similar between the treatments (22.8\%and $24.1 \%$ in the vaginal and oral group respectively), that made them do further clinical investigations [14]. By the year 2011 three randomized controlled trials $[14,17,18]$ which included 2348 patients in total which compared oral $D$ with micronized $P$ for LPS in fresh IVF cycles got included in a Cochrane review [19], that in summary said that for the outcome of clinical pregnancy, subgroup analysis of micronized $\mathrm{P}$ vs synthetic $\mathrm{P}$ showed a significant benefit from synthetic P. Conclusions could not be done on ongoing pregnancy rates or live birth rates as larger studies $[17,18]$, did not show these outcomes. In another Cochrane review higher clinical pregnancy rates were again reported with use of synthetic P in 2015 [13]. But there was a criticism regarding risk of bias of the included studies like unclear method of random sequence generation and concealing the allocation. By the year 20158 RCT [14-18,20-22] that compared oral D and either micronized vaginal P (7 comparisons having a total of 2496) or vaginal gel ( 2 comparisons, $n=1735$ ) got included in the latest systematic review and meta-analysis [23]. Oral D was given in $20-40 \mathrm{mg}$ doses while $600-800 \mathrm{mg}$ micronized $\mathrm{P} / 8 \%$ vaginal gel (crinone) got used in the control arm. Clinical pregnancy rate was higher in synthetic $\mathrm{P}$, oral $\mathrm{D}$ as compared with micronized P [relative risk [RR]1.19,95\%CI 1.04-1.36; $\mathrm{I}^{2}=6 \%$ ) an effect not seen in comparison with vaginal gel. Even though there was a large total sample size in the meta-analysis, there was risk of bias in individual studies and heterogeneity clinically in between studies like in doses that were compared, outcome reporting was incomplete, with most studies reporting only clinical pregnancy rates in most trials and insufficient safety surveillance in nearly all trials, still limited the external validity of the meta-analysis. Patki et al. [17] compared 30mg oral D with 600mg /d micronized vaginal $P$ in 675 randomized patients which suggested superiority of oral D in terms of clinical pregnancy achievement [RR 1.39,95\% CI 1.13-1.72]. Thus, that dose was decided to be used for further development and in 2013 a company sponsored phase III trial program got started, with the aim to establish the efficacy and safety of daily30mg oral D as compared with natural (clinical trial reg NoNCT01850030 and NCT02491437) for LPS in IVF cycles, with fresh embryo transfers. On completion more than 2000randomized study subjects in 2 large studies with complete assessment from start of treatment to childbirth and the child's health respectively .1st of the 2 studies namely LOTUS 1 got reported recently [24].

This was a multinational, multicentered, randomized double blind, double dummy clinical study. In 1,031 subjects undergoing IVF or ICSI with fresh single or double embryo transfer (ET) after COS were randomized on the day of oocyte retrieval into one of the treatment arms. The experimental group received oral D in 10mg tablets (Abbott), with placebo intravaginal capsules (Catalent) three times a day and the control group received micronized vaginal P 200mg capsules (uterogestan), with oral placebo tablets (Abbott)starting on the evening of the day of oocyte retrieval and discontinuing on a negative serum HCG test or at 12 gestational weeks. This study had the advantages that it was designed and powered to show noninferiority of oral D for ongoing pregnancy chances at 12 wks gestation age. Having the double-dummy design made sure that every patient received oral tablet and vaginal capsules. Hence its preference of route of administration could not me made out. This allowed to see the adverse effects without the risk of differences in nocebo between groups i.e. a self-fulfilling prophecy on expected side effects of a given drug or route of administration. Mean age of the patient was 32.5yrs in the LOTUS 1 study with mean BMI being $23 \mathrm{~kg} / \mathrm{m} 2$, with $3 \%$ of patients undergoing single embryo transfer. Thus, LOTUS1 study clearly established the noninferiority of oral D over micronized vaginal P. The ongoing pregnancy rate were $37.6 \%$ and $33.1 \%$ respectively in the oral D and micronized vaginal $\mathrm{P}$ treatment groups (difference $+4.9 \%$ with oral D;95\% CI-0.8 to+10.7\%).This single trial could not establish 
superiority at a statistically significance because of the design and sample size, which was still too small for getting a pregnancy rate difference of the magnitude of $<=5 \%$ that could be detected with confidence .On the reverse noninferiority of $600 \mathrm{mg}$ micronized vaginal $P$ against $30 \mathrm{mg}$ oral D for LPS in a fresh IVF cycle has come under the examination with the LOTUS1 trial results asthe95\% CI of the differences in ongoing pregnancy at 12 weeks includes effect sizes(-1.2 to $+10.6 \%)$ not in favor of vaginal $\mathrm{P}$, that will not be acceptable for most treating doctors.

The drug which was used for comparison in the LOTUS1 trial uterogestan is not there in USA. Uterogestan consists of $100 \mathrm{mg}$ micronized $\mathrm{P}$ in refined sunflower oil, which was previously peanut oil, soya lecithin, glycerol, titanium dioxide and purified water which is present in a soft gelatin capsule. In USA the 2 vaginal preparations of $\mathrm{P}$ for COS with respect to LPS in IVF are endometrin, which consists of an effervescent tablet made of $\mathrm{P}$ in starch (100mg micronized $\mathrm{P}$ in lactose monohydrate, polyvinyl pyrrolidone (povidone K29/32), adipic acid, sodium bicarbonate, sodium lauryl sulphate, magnesium stearate, pregelatinized maize starch and colloidal silicone dioxide). Besides that, crinone which is micronized $\mathrm{P}$ that is given in a gel form that supposedly adheres better to the vaginal wall. One dose of crinone $8 \%$ consists of $90 \mathrm{mg}$ micronized $\mathrm{P}$ in a gelof glycerol, paraffin light liquid, hydrogenated palm oil glyceride, carbonate $974 \mathrm{P}$, polycarbophil, sorbic acid, sodium hydroxide and purified water. The differences in pregnancy rates between these 2 have never been compared [13]. Oral D has been tested against crinone gel. No difference in ongoing pregnancy was found (RR0.97,95\%CI0.83-1.13), though the dose of D was only $20 \mathrm{mg}$ in both trials [23]. No randomized study compared endometrin vis a vis oral D, nor any study comparing I/M P vs oral $D$ [13]. Because of concerns of vaginal $P$ effectiveness still there is use of I/M P in USA and in lots of other parts of world.

\section{Advantages of an Oral preparation over other Routes}

In India due to social reasons use of vaginal preparation is not preferred. In questionaries' carried out by Chakraborty et al. the satisfaction of patients with tolerability of oral D for LPS in 20mg dose was markedly higher as compared with micronized vaginal P(3X200mg). Chandrashekhar et al in another RCT carried out on 831 patients who were undergoing IVF [21], significantly more satisfaction with oral $\mathrm{D}$ and dissatisfied with vaginal $\mathrm{P}$ gel when trying to rank the drugs on scale1-5. This was not found in a recent study from Iran on 240 patients where equal distribution was found between total satisfaction and dissatisfaction for oral D and 2x400mg vaginal P for LPS [22]. Thus, one needs to individualize according to patient preferences, like some who feel they are getting a much superior medicine by injectable drug or other uncomfortable modes of giving and that might influence the response to drug. Hence such preferences should be taken into consideration when choosing the drug and mode of administration. Also, when comparing oral versus vaginal misoprostol oral preparation has been preferred [25].

\section{Problem of Vaginal administration for the Treating Physician}

Normally LPS using P occurs usually within the time interval between oocyte pickup and embryo transfer. Once the ET catheter goes through the cervical canal risk of putting not only $\mathrm{P}$ itself, but also remnants of the tablets/suppositories or gel into uterine cavity are there. Moreover, the supraphysiological P concentrations in the vagina might change the local microbiome, that had become a point on which concentration is being done with relevance to IVF [26]. Though a negative effect of drug excipients or a high dose of $\mathrm{P}$ in the endometrium, embryo or the microbiome have never been documented physicians usually see to it that outer cervical canal os is cleaned before attempting the ET. Though no formal study was done it has been observed that most doctors prefer a cleaner vagina and hence prefer oral or $\mathrm{i} / \mathrm{m}$ preparation while attempting ET or a transvaginal scan at later stage.

\section{Safety of oral D}

Bioidentical orally given $\mathrm{P}$ has been associated with the formation of sedative metabolites in view of the first pass effect in the liver. They act centrally, giving the side effects of $\mathrm{P}$ like fatigue, headache and urinary frequency, besides the safety concerns related to intrahepatic cholestasis with oral preparations, prompted the development of vaginal preparations for LPS in IVF $[27,28]$. With vaginal preparation biggest problem encountered is discharge and irritation. Chakravorty et al carried out an objective examination regarding tolerability of oral $\mathrm{D}(20 \mathrm{mg})$ as compared with $600 \mathrm{mg}$ vaginal P [14]. Liver function test (LFT) was done at baseline and on d14 i.e. after 14 days of intake. The percentage of patients with abnormal LFT's and mean serum glutamate-pyruvate transaminase, bilirubin and alkaline phosphatase levels were higher between groups. In 10.5\%pts given micronized P, Vaginal discharge or irritation was confirmed, while $\%$ of D patients had those side effects. Tomic et al. [21] showed that peritoneal irritation, vaginal bleeding, discharge along with interference with sexual activity was significantly more in patients getting vaginal $\mathrm{P}$ gel as compared to oral D. No difference was seen in dizziness, headache, nausea, breast tenderness or bloating. Best insight came from the LOTUS1 trial [24] where maternal safety and tolerability regarding oral D was given. Once doctors and patients were blinded with randomization of each patient getting oral $\mathrm{D} /$ micronized $\mathrm{P}$ along with dummy medication with placebo. Also, patients got monitored for complications in Late pregnancy. $12.4 \%$ of D subject s and $16 \%$ of micronized vaginal $P$ had some adverse events at the study termination. LFT was normal in nearly all in both groups. Since there were very little side effects causing termination of the drug grouping of symptoms was done as per organ system (like gastrointestinal (GIT), nervous system, reproductive organ system, vascular system). No differences were found, and no new safety /tolerability issues were identified. Thus, Oral $\mathrm{D}$ avoids vaginal problems seen with vaginal preparations and themselves did not show any systemic tolerability differences in this large double-blind double-dummy randomized study. 


\section{Fetal Safety}

D Since 1960's after its manufacture in 1950's has been in the market for use in pregnancy with the indication of recurrent abortion or threatened miscarriage in multiple countries worldwide with a total cumulative exposure of 26 million patient years. From the sales figures it was estimated that more than 8million fetuses must have been exposed in utero to $\mathrm{D}$, with greater than half a century of use globally [29]. This extensive use theoretically rules out any substantial fetal risk. Yet low level risk can be detected only by a sophisticated and large observational study. From the available data along with analysis in depth 28 cases of congenital defects attributable potentially to D exposure in pregnancy from 1977 to 2005, got recorded [30]. Malformation rates which are associated with a drug can't be calculated using pharmacovigilance data and the low number of reported cases, few occurring within controlled studies in relation to the estimated number of pregnancies exposed suggests a relative teratogenic risk of D is very unlikely. Also, the kinds of defects attributed to D were very diverse, not having any pattern of abnormalities [31]. In the LOTUS 1 Trial, there was a recording of child health at birth for the total maternal population and 6 months after birth in a subset of 216 patients, that were treated in Russia. In total 213 and 158 children were observed in the oral D and vaginal $\mathrm{P}$ group respectively. The incidence of congenital, familial and genetic disorders was under2\% I both the groups. Neither any difference in congenital defect incidence was there nor any pattern of congenital malformations with either the use of D or P.

More data was retrieved from the RCT's on use of D in threatened miscarriage [32-36] and recurrent miscarriages [37], no safety concerns were revealed over D use. A retrospective study that was a case control study, a comparison of 202 children who were born with congenital heart disease and had received exposure to D during pregnancy, were compared with 200 control group of healthy children who were born from 2010 to 2013in the Gaza strip of Palestine [38]. D exposure was any recall of D use in the first trimester of pregnancy. A greater incidence of D intake was found in mothers of children with a heart defect (38\%) as compared with control children (18\%) and hence Zaquot et al. [38] concluded that a positive correlation between D use during early pregnancy and congenital heart disease in the offspring (adjusted odds ratio 2.71, 95\% CI1.54-4.24; $\mathrm{P}<0.01$ ). Yet there were a lot of violations of basic principles of epidemiological research:

i. The comparisons were required within same study base namely women having indication for $\mathrm{D}$ and those who received it or not.

ii. Since D is commonly used for prevention of miscarriage; all women are required to have same background, getting differences in maternal population add confounding factors. Literature states that women at risk or with history of earlier abortions have a strong risk for developing congenital heart disease [39-41]. iii. Zaquot et al did not confirm even retrospectively the intake of $\mathrm{D}$ and just relied on mothers recalling the same. Normally mothers recall anything that occurred during pregnancy once they had a child with a congenital abnormality.

iv. Various heart defects got pooled in one group, and socioeconomic status got ignored. Thus, from this study conclusions cannot be drawn regarding relationship of D Causing congenital heart defects.

Normally incidence of congenital cardiac defects is $1 \%$. To verify or refute this hypothesis of CHD incidence following D exposure in offspring would need over 3000 infants get studied at 1:1 randomized trial. Given a live birth rate of $30 \%$ in patients who undergo IVF, a two armed study on women getting $\mathrm{D}$ or a control drug for LPS would need a total sample of $>10,000$ patients (alpha error $<5 \%$,beta error $<20 \%$ ).It is not likely that a study of such a huge dimension will be done soon, and hence physicians /treating ivf specialists will have to rely on available data from pharmacovigilance. Large sized randomized studies comparing risk of bioidentical P have not been done, inspite of risk theoretically of bioidentical P in supra physiological doses can't be ruled out.

\section{Cost Effectiveness of $\mathrm{D}$}

For checking cost effectiveness of any drug, one needs to compare its efficacy and cost with a comparable drug. Griesinger et al. conducted this for 2 countries namely Russia and China in an economic model which used live birth as the primary efficacy outcome, along with direct comparison of cost of D (Duphaston) vs micronized $\mathrm{P}$ (uterogestan)along with comparing the infertility treatment costs $[42,43]$. In both places, a lower cost/live birth was seen with the use of $D$.

\section{Conclusions}

Just like Chakravorty et al. we have been proponents of use of D for LPS in India in view of the social reasons comparing D with I/M micronized progesterone in oocyte donation cycles from the early 2000's [44,45]. We also possibly had a bias in our studies not using the double dummy design. With the LOTUS 1 trial where large double blind, double-dummy phase trial which compared $30 \mathrm{mg}$ oral D for LPS in IVF, efficacy findings of multiple previous independent trial gets confirmed and establishes the noninferiority of oral $30 \mathrm{mg}$ oral D vs daily $600 \mathrm{mg}$ micronized vaginal P. Oral D was well tolerated inspite of first pass through liver. Since most women prefer the oral preparation, D might become the new standard of care for LPS in fresh embryo transfer IVF cycles, with it being the cheaper preparation as well and no new concerns of fetal safety.

\section{References}

1. Hoffmann V, Pedersen PA, Philiip J, Fly P, Pedersen (1988) C The effect of dydrogesterone on premenstrual symptoms. A double blind,randomized ,placebo controlled study in general practice. Scand J Prim Health Care 6(3): 179-83.

2. Brown J, Kives S, Akhtar M M (2012) Progestogens for pain associated with endometriosis. Cochrane Database Syst Rev Progestogens for pain associated with endometriosis. Cochrane Database Syst Rev 14(3). 
3. Lee HJ, Park TC, Kim JH, Norwitz E, LeeB (2017) The influence of oral dydrogesterone and vaginal progesterone on threatened abortion: A systematic review and meta-analysis. Biomed Res Int p.10.

4. Saccone G, Schoen C, Franasiak JM, ScottRT Jr, Beghella V (2017) Supplementation with progestogens in the first trimester of pregnancyto prevent miscarriage in women with unexolained recurrent miscarriage: Ac systematic review and meta-analysis of randomized controlled trials. Fertil Steril 107(2): 430-438.

5. Stevenson JC, Panay N, Pexman-Fieth C (2013) Oral esradiol and dydrogesterone combination therapy in pistmenopausal women: Review of efficacy and safety. Maturitas 76(1):10-21.

6. Schindler AE, Campagnoli C, Druckmann R, Huber J Psqualini JR, et al. (2013) Classification and pharmacology of progestins. Maturitas 46(Suppl 1): S7-S16.

7. Kuhl H (2005) Pharmacology of estrogens and progestogens: Influence of different routes of administration. Climacteric 8(Suppl1): 3-63.

8. Rizner TL, Brozic P, Doucette C, Turek-Etienne T, Muller-Vieira U, et al. (2011) Selectivity and potency of of the retroprogesterone dydrogesterone in vivo. Steroids 76(6): 607-615.

9. Yu S, Long H, Chang HY, Liu Y, Giao H, Zhu J, et al. (2018) New applications of dydrogesterone as a part of progestin -primed ovarian stimulation protocol for IVF:a randomized controlled trial including 516 first IVF/ ICSI cycles. Hum Reprod 33(2): 229-237.

10. Beranic N, Gobec S, Rizner TL (2011) Progestins as inhibitors of the human 20ketosteroid reductases, AKR1C1and AKR1C3. Chem Biol Interact 191(1-3): 227-33.

11. (2015) Practice Committee of the American Society of Reproductive Medicine. Current clinical irrelevance of luteal phase deficiency: A committee opinion. Fertil Steril103: e27-32.

12. Fatemi HM, Popovic-Todorovic B, Papanikolau E, Donoso P, Devroey P (2007) An update on luteal phase support in stimulated IVF cycles. Hum Reprod Update 13(6): 581-90.

13. Van der Linden M, Buckingham K, Farquhar C, Kremer JA, Metwally M (2015)Luteal phase support for assisted reproductive cycles. Cochrane Database Syst Rev.

14. Chakravarty BN, Shirazee HH, Dam P, Goswami SK, Chatterjee R (2005) Oral dydrogesterone versus intravaginal micronized progesterone as luteal phase supportin assisted reproductive technology(ART) cycles: Results of a randomized study. J Steroid Biochem Mol Biol 97(5): 416420.

15. Chakravarty BN, Dam P, Goswami SK, Ghosh S, Chattopadhya R (2005) Oral dystogesterone versus intravaginal micronized progesterone as luteal phase support in ART cycles-evaluation based on hormonal profile and clinical outcome. Hum Reprod.

16. Chakravarty BN, Sharma S, Ghosh S, Chattopadhya R, Goswami SK (2006) Oral dydrogesterone versus intravaginal micronized progesterone as luteal support in ART cycles: Evaluation based on hormonal profile and clinical outcome. Hum Reprod.

17. Patki A, Pawar VC (2007) Modulating fertility outcome in assisted reproductive technology by the use of dydrogesterone. Gynecol Endocrinology 23(Suppl1): 68-72.

18. Ganesh A, Chakravorty N, Mukherjee R, Goswami S, Chaudhary K, et al. (2011) Comparison of oral dydrogesterone with preogesterone gel and micronized progesterone for luteal support in 1373 womenindergoing in vitro fertilization: A randomized clinical study. Fertil Steril 95(6): 1961-1965.

19. Vander Linden M, Buckingham K, Farquhar C, Kremer JA, Metwally M (2011) Luteal phase support for assisted reproductive cycles. Cochrane Database Syst Rev.
20. Salehpour S, Tamimi M, Sahar khitz N (2013) Comparison of oral dydrogesterone versus vaginal progesterone gel in. Luteal phase support in vitro fertilization cycles. Iran J Reprod Med 11(11): 913-918.

21. Tomic V, Tomic J, Klaic DZ, Kasum M, Kuna K (2015) Oral dydrogesterone versus vaginal progesterone gel in Luteal phase support: Randomized controlled trial. Eur J Obstet Gynecol Reprod Biol 186: 49-53.

22. Saharkhiz N, Zamaiyan M, Salehpour S, Zadeh modarres S, Hoseini S, et al. (2016) A comparative study of oral dydrogesterone and micronized progesterone for Luteal phase support during in vitro fertilization cycles. Gynecol Endocrinol 32(2): 213-217.

23. Barbosa MW, Silva LR, Navarro PA, Ferriani RA, Nastri CO (2016) Dydrogesterone versus progesterone for luteal phase support: A systematic review and meta-analysis of randomized controlled trials. Ulrasound Obstet Gynecol 48(12): 161-70.

24. Tourmaye H, Sukhikh GT, Kahler E, Griesinger G (2017)A phase III randomized control trial comparing the efficacy, safety and tolerability of oral dydrogesterone versus micronized progesterone for luteal phase support in in vitro fertilization. Hum Reprod 32(5): 1029-1027.

25. Ashok PW, Hamoda H, Nathani F, Flett GM, Templeton A (2003) Randomized controlled study comparing oral and vaginal misoprostol for preabortion cervical priming prior to surgical termination of pregnancy.

26. Garcia-Velasco JA, Menabrito M, Catalan IB (2017) What fertility specialists should know about the vaginal microbiome:areview. Reprod Biomed Online 35(1): 103-112.

27. Bacq Y, Sapey T, Brechot MC, Pierre F, Fignon A, Dubois F (1997) Intrahepatic cholestasis of pregnancy: a French prospective study. Hepatology 26(2): 358-364.

28. De Ziegler D, Fanchin R (2000) Progeterone and progestins: applications in gynaecology. Steroids 65(1): 671-679.

29. Medicines and Healthcare products Regulatory Agency (MHRA) (2018) MHRA UK public assessment report. Efficacy of progestogens in the maintenance of early pregnancy in women with threatened miscarriage or recurrent miscarriage. feb14.

30. Queisser-Luft A (2009) Dydrogesterone use during pregnancy: overview of birth defects reported since 1977.Early Hum Dev 85(6): 375-377.

31. Sukhikh GT, Baranov IL, Meichenko GA, Bashmakova CV, Blockeel C, et al. (2017) A phase III randomized controlled trialof Oral dydrogesterone versus micronized vaginal progesterone for lutealphasespport in in vitro fertilization, with focus on the Russian population. Akush Ginekol (Mosk) 32(5): 1019-1027 Russia.

32. El Zibdeh MY, Yousef LT (2009) Dydrogesterone support in threatened miscarriage. Mauritas 65(1): 543-546.

33. Pandiyan RU (2009) Dydrogesterone in threatened miscarriage: a Malaysian experience. Mauritas 65(1): 547-550.

34. Ehrenskjoeld ML, Bondo B, Welle F (1967) Treatment of threatened abortion with dydrogesterone. Ugeskr Laeger 129(50): 1678-1679.

35. Misto A (1967) Experiences with 6-dehydro-retopogesterone I the treatment of placental insufficiency. Ann Obstet Ginecol Med Perinatal 89: 102-112.

36. Vincze E, Molnar GB, Foldesi I, Pal A (2006) Treatment possibilities for threatened abortion using progesterone and progesterone type drugs. Magyar NoorvosokLapja 69: 281-284.

37. El Zibdeh MY (2005) Dydrogesterone in the reduction of recurrent spontaneous abortion. J Steroid Biochem Mol Biol 97(5): 431-434.

38. Zaqout M, Aslem E, Abuqamar M, Abughazza 0, Panzer J, et al. (2015) The impact of oral intake of Dydrogesterone on fetal heart development during early pregnancy.Pedatr Cardiol 36(7):1483-1488. 
39. Tikkanen J, Heinonen OP (1992) Congenital heart disease in the off spring and maternal habits during and home exposure during pregnancy. Teratology 46(5): 447-454.

40. LiuS, Liu J, Tang J, JiJ, Chen J, Liu C (2009) Environmental risk factors for Congenital heart disease in the Shandong Penensula, China:a hospital based case control study. J Epidemiol 19(3): 122-130.

41. Shi H, Yang S, Liu Y, Huang P, Lin N, et al. (2015) Study on Environmental causes of SNPs of MTHFR, MS and CBS related to congenital disease. PLoS One.

42. Griesinger G, Tournaye H, Connolly MP, Pexman-Fieth C (2017) A comparison of live birthrates and cost effectiveness analyses in luteal support based on a multicenter, double blind RCT of oral dydrogesterone vs micronized vaginal progesterone. Asia Pacific Initiative on Reproductive Medicine (ASPIRE 2017), Kuala Lumpur, Malaysia.
43. Griesinger G, Tournaye H, Sukhikh GT, Kalinina E, Abubakirov A, et al. (2017) Comparing the cost per livebirth for treatment of luteal support in Russia based on a multicenter,double blind RCT of oral dydrogesterone vs micronized vaginal prgeserone. Mother and Child. XVIIIth All Russia Scientiific Forum, Modcow.

44. Allahbadia GN, Kaur KK, et al. (2004) The comparison of pregnancy outcomes of intramuscular progesterone versus oral dydrogesterone for luteal phase support in donor egg IVF recipient cycles coauthor Fertil Steril 1: 171.

45. Kaur Kulvinder K (2006) Role of Dydrogesterone in Luteal phase Support and recurrent Abortion, an update on pharmacology of dydrogestrone. Accepted for oral presentation for CORI Conference to be held in Spainin -got postponed.

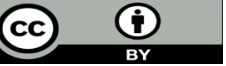

This work is licensed under Creative Commons Attribution 4.0 License

Submission Link:

Submit Article

DOI: $10.32474 /$ OAJRSD.2018.01.000119

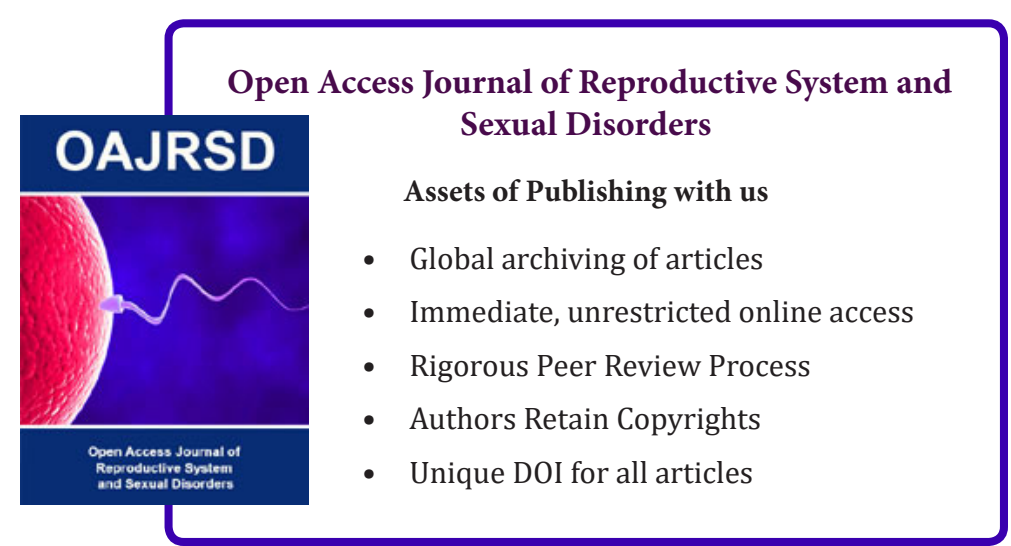

